\title{
Functional Development of Large-Scale Sensorimotor Cortical Networks in the Brain
}

\author{
Charles Quairiaux, ${ }^{1}$ Pierre Mégevand, ${ }^{2}$ Jozsef Z. Kisss ${ }^{1}$ * and Christoph M. Michel ${ }^{1,2 *}$ \\ ${ }^{1}$ Faculty of Medicine, Department of Fundamental Neurosciences, University of Geneva, 1211 Geneva, Switzerland, and ${ }^{2}$ Department of Neurology, Geneva \\ University Hospital, 1211 Geneva, Switzerland
}

\begin{abstract}
Large-scale neuronal networks integrating several cortical areas mediate the complex functions of the brain such as sensorimotor integration. Little is known about the functional development of these networks and the maturational processes by which distant networks become functionally connected. We addressed this question in the postnatal rat sensorimotor system. Using epicranial multielectrode grids that span most of the cortical surface and intracortical electrodes, we show that sensory evoked cortical responses continuously maturate throughout the first 3 weeks with the strongest developmental changes occurring in a very short time around postnatal day 13 (P13). Before P13, whisker stimulation evokes slow, initially surface-negative activity restricted mostly to the lateral parietal area of the contralateral hemisphere. In a narrow time window of $\sim 48 \mathrm{~h}$ around P13, a new early, sharp surface-positive component emerges that coincides with subsequent propagation of activity to sensory and motor areas of both hemispheres. Our data show that this new component developing at the end of the second week corresponds principally to functional maturation of the supragranular cortical layers and appears to be crucial for the functional associations in the large-scale sensorimotor cortical network. It goes along with the onset of whisking behavior, as well as major synaptic and functional changes within the $S 1$ cortex that are known to develop during this period.
\end{abstract}

\section{Introduction}

The complex functions of the cerebral cortex are thought to rely on widely distributed and intermingled cortical networks of different scales working together to process sensory, motor, or cognitive tasks. The sensorimotor system of rodents represents a simple model of large-scale networks. Unilateral whisker stimulations trigger evoked activity in the contralateral S1 cortex (Welker, 1976; Armstrong-James and Fox, 1987) and in other areas, such as the $\mathrm{S} 1$ cortex in the opposite hemisphere and the secondary somatosensory and frontal motor cortices in both hemispheres (Shuler et al., 2001; Benison et al., 2007; Ferezou et al., 2007; Mégevand et al., 2008). Such interactions are thought to be important for spatial correlation of inputs and sensorimotor integration (Ahissar and Kleinfeld, 2003; Kleinfeld et al., 2006). At what time this sensorimotor network becomes functional during development remains unknown.

In rodents, cortical neurogenesis, cell migration, and layer and area patterning are almost complete at birth or early after (Sur

Received Nov. 15, 2010; revised April 20, 2011; accepted May 17, 2011.

Author contributions: C.Q., J.Z.K., and C.M.M. designed research; C.Q. and P.M. performed research; C.Q. analyzed data; C.Q. wrote the paper.

*J. Z. Kiss and C. M. Michel contributed equally to this work as senior authors.

This work was supported by Swiss National Foundation Grant31003A_130781/1 to J.Z.Kand 310030_132952 to C.M.M., by the Swiss National Foundation Special Program University Medicine (33CM30-124101, and 33CM30124089 to (.M.M. and J.Z.K.), and by the Von Meissner Foundation to C.Q. and J.Z.K. The Cartool software was programmed by Denis Brunet, supported by the Center for Biomedical Imaging of Lausanne and Geneva (CIBM). We thank C. Saadi for technical assistance.

Correspondence should be addressed to Professor Christoph M. Michel, Neufo-CMU, 1211 Genève 4, Switzerland. E-mail: Christoph.michel@unige.ch.

DOI:10.1523/JNEUROSCI.5995-10.2011

Copyright $\odot 2011$ the authors $\quad 0270-6474 / 11 / 319574-11 \$ 15.00 / 0$ and Leamey, 2001; Price et al., 2006; Rash and Grove, 2006). Segregation of thalamocortical (TC) terminals and their layer IV neuronal partners in barrel structures takes place during the first 5-6 d (Erzurumlu and Jhaveri, 1990; Schlaggar and O'Leary, 1994). By P7, cortical neurons are set in their final layers; however, their morphology and synaptic organization is still immature (Foeller and Feldman, 2004). Growth of tangential connections within infragranular and supragranular layers of the S1 cortex has already begun but continues until the third week (Miller et al., 2001; Dagnew et al., 2003; Sehara et al., 2010). This protracted development correlates with extended plasticity in layers II/III (Fox, 2002). Similarly, ipsilateral and callosal longrange projections from $\mathrm{S} 1$ reach their targets during late embryogenesis and early postnatal development, but refinements of those projections continue for the entire postnatal period (Wise and Jones, 1978; Ivy and Killackey, 1982; Price et al., 2006). Although studies have been conducted on the morphological development of local and long-range connectivity of the S1 cortex, the dynamics of the functional maturation of the sensorimotor networks at the large-scale level have not yet been investigated. To explore this development, we recorded here multichannel epicranial somatosensory evoked potentials (SEPs). In addition to conventional voltage analysis, we applied spatiotemporal mapping methods to describe the propagation of evoked activity across the brain surface (Michel et al., 2004). This approach was complemented by intracortical local field potential (LFP) recordings to confirm the localization of cortical generators and to study the development of laminar cortical responses in areas selected from the surface recordings. LFP recordings were analyzed using current-source density (CSD) and multiunit activity (MUA). 
Our results revealed profound changes in cortical response patterns and show that the recruitment of the large-scale sensorimotor networks by whisker stimulation is set at the end of the second week, initiated by the abrupt appearance of an early sharp SEP component. The postnatal period in rat is an ideal model for studying the cortical development processes that take place in the late fetal stages and early postnatal period in humans (Clancy et al., 2001).

\section{Materials and Methods}

A total of 65 Wistar rats were used in this study, equally distributed between male and female. Animal handling procedures were approved by the Office Vétérinaire Cantonal (Geneva, Switzerland) in accordance with Swiss Federal Laws.

\section{Electrophysiological recordings}

Anesthesia. Anesthesia was initiated in a box ventilated with isoflurane at $2.5 \%$ in $20 \% \mathrm{O}_{2} / 80 \%$ air. As previously described (Quairiaux et al., 2010), rats were then mounted in a stereotaxic frame providing a continuous flow of isoflurane in the same air mixture. Body temperature was maintained at $37^{\circ} \mathrm{C}$ with a homeothermic heating blanket. A subcutaneous dose of bupivacaine was injected above the skull before the skin was incised on the midline from the frontal to the occipital pole and retracted laterally. During all surgical procedures, isoflurane concentration was maintained at $2.5 \%$, while it was lowered to the minimal level, preventing the hindlimb withdrawal reflex $(0.7-1.1 \%)$, during recordings. Furthermore, we monitored the physiological status of the animals by keeping the respiratory rate at $60-80$ per second.

Epicranial and intracortical recordings. Recording procedures were detailed previously (Mégevand et al., 2008; Quairiaux et al., 2010). Differential potentials were recorded with a custom-made amplifier (gain $5000 \times)$ and digitally converted (A/D converter DT3004, Data Translation) using custom-made scripts in VEE Pro 6 (Agilent). For epicranial and intracortical LFP recordings, potentials were bandpass filtered between 1 and $500 \mathrm{~Hz}$ and sampled at $2 \mathrm{kHz}$. For simultaneous recordings of LFPs and MUAs, the signal was recorded on a wider bandpass (1-2000 $\mathrm{Hz}$ ) and sampled at $6250 \mathrm{~Hz}$, then separated by off-line digital filtering between 1 and 500 and between 750 and $2000 \mathrm{~Hz}$, respectively (see below, Data analyses).

Epicranial SEPs were recorded from arrays of 16 stainless steel electrodes $500 \mu \mathrm{m}$ in diameter dipped in EEG paste (EC2, Grass Technologies; final impedance $\approx 50 \mathrm{k} \Omega$ ). Two different arrays were used to adapt to the growth of the animal and to cover the entire skull surface. Electrode coordinates from bregma for the right and left hemispheres were (rostrocaudal/mediolateral; see Fig. $1 \mathrm{~A}$ ): from P7 to P14, $-6 \mathrm{~mm} / 4 \mathrm{~mm}$ (e1, e15), $-3.5 / 5$ (e2, e14), -3/2.25 (e3, e13), - 1/5 (e4, e12), -0.25/2.25 (e5, e11), 1.25/4 (e6, e10), 3/2 (e7, e9), 1/0 (e8), -5/0 (reference), ground electrode at 5.5/0; from P16 to P21, $-7.5 \mathrm{~mm} / 4 \mathrm{~mm}$ (e1, e15), $-4.75 / 5$ (e2, e14), -3.5/2.25 (e3, e13), -1.5/5 (e4, e12), -0.75/2.25 (e5, e11), $1.25 / 4$ (e6, e10), 3.25/2.25 (e7, e9), 0/0 (e8), $-6.25 / 0$ (reference), ground electrode at $6 / 0$. At the end of the recording sessions, animals were either killed with an intraperitoneal injection of sodium pentobarbital or processed for intracortical recordings, or allowed to recover for $24 \mathrm{~h}$ before another epicranial SEP recording session was processed. In this last case, the skull was cleaned with saline and the surgical wound sutured before returning the rat to its cage.

For intracortical recordings, separate large craniotomies were performed in the right parietal and frontal bones to expose the frontal and lateral parietal cortices. The surface of the brain was covered with sterile saline solution heated at $37^{\circ} \mathrm{C}$. Recordings were made in the two regions successively with a linear 16-electrode probe (iridium based, $177 \mu \mathrm{m}^{2}$ diameter, $100 \mu \mathrm{m}$ interelectrode spacing; NeuroNexus Technologies) inserted through the dura mater perpendicular to the cortical surface; the reference electrode was attached to the skin. Recordings ranged from (rostrocaudal/mediolateral) 2.0 to $3.2 / 1.5$ to $2.0 \mathrm{~mm}$ for frontal cortex and -1.0 to $-2.5 / 4.5-5.5 \mathrm{~mm}$ for parietal cortex.

Histology. The positioning of the recording sites in the parietal cortex relative to barrels was checked histologically by performing Chicago Sky
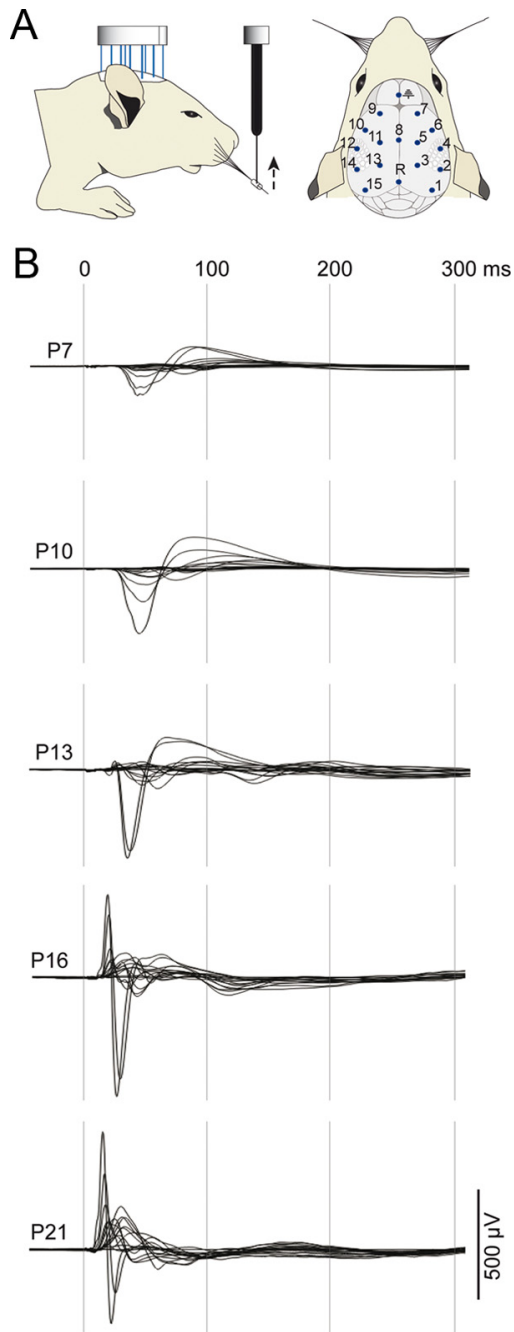

Figure 1. Postnatal development of somatosensory evoked potentials in response to whisker deflection from P7 to P21. A, Design of the epicranial recording setup. Under isoflurane anesthesia, a multielectrode grid is placed in contact with the skull and SEPs are recorded in response to upward deflections of all whiskers on one side of the face. The position of the electrodes on the skull is schematized on the right diagram. $\boldsymbol{B}$, Superimposed grand average SEPs from all electrodes recorded at P7 $(n=12), \mathrm{P} 10(n=11), \mathrm{P} 13(n=11), \mathrm{P} 16(n=7)$, and P21 $(n=13)$, illustrating the progressive modifications of the SEP waveforms during the postnatal period.

Blue Dye iontophoretic injection ( $20 \mu \mathrm{A}, 8 \mathrm{~s}, 1000 \mu \mathrm{m}$ depth) with a glass microelectrode at the end of the experiment. After killing and perfusing the rats with $4 \%$ paraformaldehyde, tangential sections of the cerebral cortex were processed for cytochrome oxidase (CO) staining as previously described (Quairiaux et al., 2010). Briefly, the cortex was isolated from the subcortical structures, rinsed in phosphate buffer ( $\mathrm{pH} 7.4)$, cryoprotected in $30 \%$ sucrose- $0.9 \% \mathrm{NaCl}$, and flattened between two glass slides during snap freezing. Serial sections $(40 \mu \mathrm{m})$ were cut on a cryostat, rinsed in $0.1 \mathrm{M}$ phosphate buffer ( $\mathrm{pH} 7.4)-4 \%$ sucrose, and exposed for $3 \mathrm{~h}$ at $37^{\circ} \mathrm{C}$ to CO: $0.075 \%$ cytochrome $c$ (Sigma C 2506), $0.04 \% 3,3^{\prime}$-diaminobenzidine (Sigma), and $0.1 \mathrm{~m}$ phosphate buffer- $4 \%$ sucrose. Sections were then mounted on gelatin-coated slices. Electrode positions were always found between rows $\mathrm{C}$ and $\mathrm{E}$ and arcs $1-4$ of the barrel cortex. The cortical layer borders in the motor frontal and sensory parietal regions (see Figs. 4-8) were estimated on the basis of cytoarchitecture on Nissl-stained coronal sections made in a subset of the P10 and P21 animals. After cryoprotection as described above, coronal sections were cut, mounted on gelatin-coated slices, and stained with cresyl violet. Linear shrinkage of the cortex was estimated to $10 \%$ from dye injections at known depths. Layer border depths were then corrected from this estimation. 
Whisker stimulation. Unilateral stimuli were delivered through a solenoid-based stimulator device simultaneously to all large whiskers (Troncoso et al., 2000). Each stimulus consisted of $500 \mu \mathrm{m}$ back-and-forth deflections with $1 \mathrm{~ms}$ rise time. Right-sided and left-sided series of 50 stimuli were applied. During preliminary experiments (data not shown), we tested different repetition rates of whisker stimulation. At P7, we observed that with 1-5 s interstimulus intervals (ISIs), the amplitude of the epicranial or intracortical voltage responses in the contralateral S1 cortex declined rapidly at subsequent deflections within a train, suggesting a steady-state adaption of the responses (Ahissar et al., 2000). For longer ISIs, the amplitude of the responses did not vary within a train. This adaptation was less pronounced in older animals. To prevent adaptation of the responses between stimuli, we set a 9 s ISI. In control experiments (data not shown), no auditory evoked activity was elicited by activating the stimulator next to the whiskers without touching them.

\section{Data analyses}

All analyses were performed using Cartool software by Denis Brunet (http://brainmapping.unige.ch/cartool) and Matlab toolboxes (MathWorks). SEP, LFP, and MUA were calculated off-line by averaging responses, $100 \mathrm{~ms}$ prestimulus to $500 \mathrm{~ms}$ poststimulus. Before MUA averaging, the recorded signal was first preprocessed with an envelope filter: 750-2000 $\mathrm{Hz}$ bandpass, second-order Butterworth filters, followed by an absolute rectification and a $1 \mathrm{~ms}$ sliding window average. Using a 16electrode linear probe, two intracortical recordings were necessary to span the cortical thickness: the uppermost electrode was first placed at the cortical surface; then, the probe was lowered $500 \mu \mathrm{m}$ deeper, with the lowermost electrode reaching $2000 \mu \mathrm{m}$ depth. Superficial and deep LFPs or MUAs were combined using a linearly weighted average for the depths at which two recordings had been obtained (Mégevand et al., 2008). Grand averages were obtained by averaging individual SEPs, LFPs, and MUAs by age groups.

Peak and onset latency analyses. Peak-topeak amplitudes were calculated as maximum positive minus negative poststimulus values. Absolute onset latencies at selected epicranial or intracortical electrodes were determined as the first time point at which the voltage value was $>4$ times the SD of its prestimulus $50 \mathrm{~ms}$ baseline for at least 5 consecutive time frames ( $2.5 \mathrm{~ms})$. For between-age group comparisons, ANOVA and Games-Howell post hoc tests (SPSS Inc.) were used. All amplitude and latency values are mean \pm SEM.

Point-wise paired statistics. To determine periods of evoked significant epicranial or intracortical signal, considering all electrodes together, we compared poststimulus to prestimulus activity with paired $t$ tests across animals and Bonferroni corrections for the number of electrodes. At each time frame of each electrode, poststimulus voltage values were compared with the $50 \mathrm{~ms}$ prestimulus average voltage of the same electrode. Evoked voltage deflections were declared significant when $p$ remained $<0.01$ for at least 5 consecutive timeframes $(2.5 \mathrm{~ms})$.

Topographic analyses of epicranial potentials. A $k$-means clustering analysis method was used to characterize the spatiotemporal dynamics of whisker-evoked activity as detailed previously (Michel et al., 2004; Mé- gevand et al., 2008; Quairiaux et al., 2010). This method allows determination of periods of stable map topographies in the grand average SEP, spatiotemporally defining the dominant steps of cortical processing of the stimulus. To evaluate the stability of the maps identified by the clustering analyses as well as to calculate their onsets and durations, each map was fitted back to SEP of individual rats. Each time point of the response of individual rats was attributed to the component map with the highest spatial correlation, a measure of the topographical similarity between two maps (Mégevand et al., 2008). The first time point of occurrence of a component map determined its onset latency; the number of time points allocated to this map defined its duration. Grand average map series are illustrated as colored segments on the global field power (GFP), calculated as the spatial SD of all voltage values at each time point and representing the strength of the electrical field.

Current-source density calculation. One-dimensional CSDs are calculated as the product of the second spatial derivative of the electric potential along the cortical depth with the conductivity tensor. Since the 
relatively small conductivity variations of different cortical depths only slightly affect CSD estimates, the conductivity tensor was assumed to be constant and CSDs were then estimated as the finite-difference second spatial derivative of the LFP using the following formula: CSD $=-[V(z+$ $\Delta z, t)-2 V(z, t)+V(z-\Delta z, t)] / \Delta z^{2}$, where $V(z, t)=$ measured voltage at subpial depth $z, t=$ time, $\Delta z=100 \mu \mathrm{m}$ (Mitzdorf, 1985). To compute the CSD at the upper extremity of the probe, virtual voltage values were extrapolated by assuming no voltage decay above the uppermost intracortical electrode. After CSD calculation, the upper and lower electrodes are lost. To better visualize the CSD profiles, color-coded plots were computed using linear interpolation.

\section{Results}

Postnatal maturation of the somatosensory evoked potentials In the first series of experiments, epicranial SEPs were recorded at 5 different postnatal days in different animals: 12 animals at P7, 11 at P10, 11 at P13, 7 at P16, and 13 at P21. As illustrated in Figure 1, SEPs undergo considerable changes: modified waveform morphologies, increase in amplitude, and decrease in onset latency and duration. The onset is always recorded at the lateral electrodes of the parietal region contralateral to the stimulus, corresponding to the $\mathrm{S} 1$ area. The ANOVA shows a significant effect of age $(F([$ df-between $=4]$, [df-within $=49])=182.2 ; p<$ $0.001)$; the onset latencies decrease from $26.9 \pm 0.4 \mathrm{~ms}$ at P7 to $6.6 \pm 0.4 \mathrm{~ms}$ at P21, with $>50 \%$ of reduction between $\mathrm{P} 13$ and P16 (22.2 $\pm 0.8 \mathrm{~ms}$ to $10.2 \pm 0.6 \mathrm{~ms}$; Games-Howell tests at $p<$ 0.001 ). The maximal peak-to-peak amplitude recorded at the same S1 electrode significantly increases from $277 \pm 28 \mu \mathrm{V}$ to $1098 \pm 115$ $\mu \mathrm{V}$ between P7 and P21 (ANOVA, $F_{(4,49)}=21.5 ; p<0.001$ ), with a twofold increase occurring between P13 and P16 $(498 \pm 199 \mu \mathrm{V}$ and $1089 \pm 111 \mu \mathrm{V}$; Games-Howell tests at $p<0.001)$.

\section{Maturation of the large-scale sensorimotor cortical network from P7 to P21}

During the postnatal period, changes were not observed solely at the $\mathrm{S} 1$ level but also in the larger-scale sensorimotor networks. To illustrate these changes, we first studied the global evolution of evoked activity at all epicranial electrodes by comparing poststimulus SEP voltage values to prestimulus voltages with multiple point-wise paired $t$ tests across subjects in the different age groups. As illustrated in Figure 2, periods of significant evoked voltages are almost entirely restricted to the contralateral parietal electrodes comprising the S1 area at P7 and P10 (Fig. 2, multiple point-wise paired $t$ tests with Bonferroni correction, $p<0.01$ for minimum 5 consecutive timeframes). The first responses are of negative voltages. Note that onset latencies of significant periods are later than those of the evoked voltages calculated at single electrodes presented above. Here, the initial onsets of the responses are missed due to the threshold induced by the statistics . Prominent changes occur at P16 and P21 compared with younger ages, with evoked responses invading numerous electrodes in both hemispheres. Moreover, the initial surface response in the contralateral S1 region that exhibited a negative polarity in younger animals now shows a positive polarity.

These analyses clearly bring to light the spreading of evoked activity across more and more surface electrodes during the postnatal period. To characterize the maturation of the spatiotemporal dynamics of the responses across the large-scale sensorimotor networks, we performed topographical analyses based on the same data to define the sequence of sensory evoked component maps in the SEP at each age group (Fig. 3). At P7 and P10, the pattern of evoked activity is summarized in two SEP maps that appear very similar at both ages in terms of topographies, onsets, and durations. The first map shows a focal negativity in the pari-
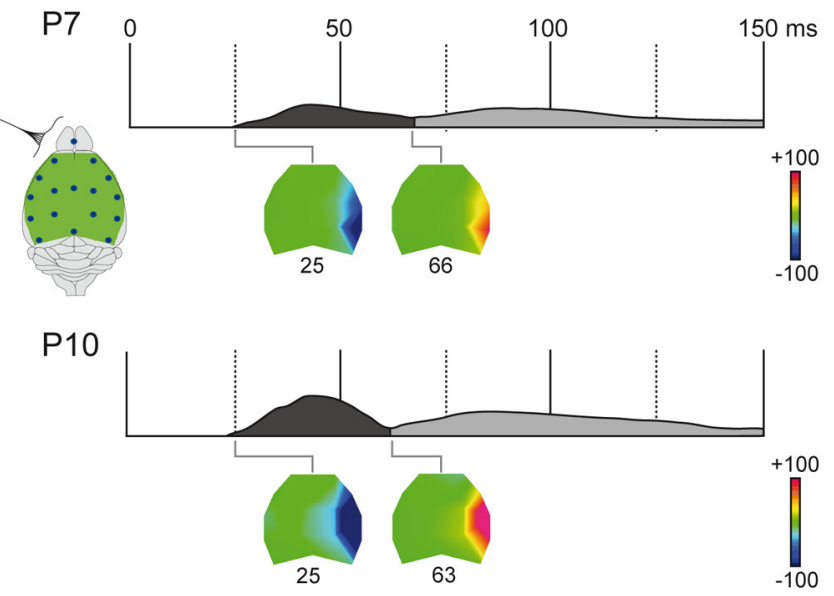

P13

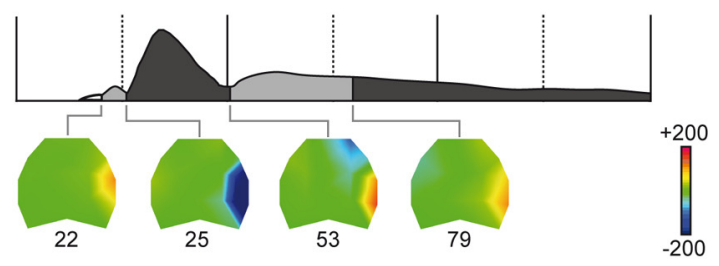

P16

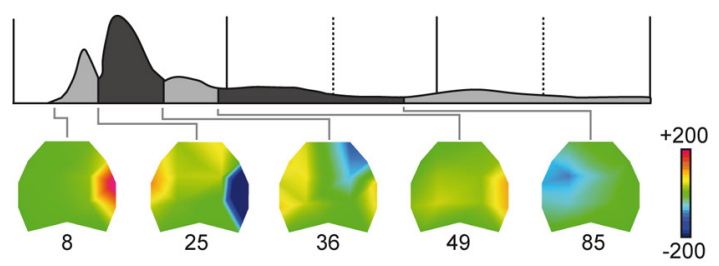

P21

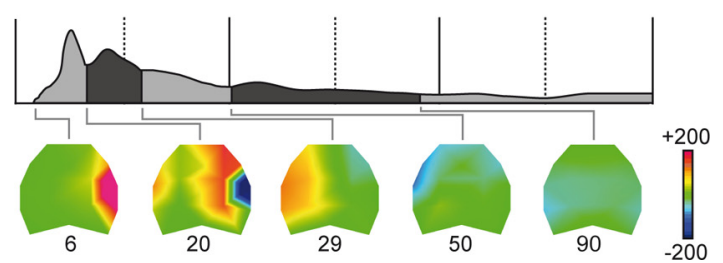

Figure 3. Postnatal development of SEP topographies. Segments identified by $k$-mean clustering segmentations indicated on the grand average GFP and corresponding mean topographic maps. Maps were calculated as average potential values during the segment period at each electrode and interpolated with Delaunay triangulations for graphical representations. Mean onset latencies below each map were calculated by fitting back individual SEPs to the clustered maps. Color scaling in microvolts is indicated at each age.

etal cortex contralateral to the stimulation at 25 ms mean onset, lasting until $\sim 65 \mathrm{~ms}$ and reversing to positive during the second map. In agreement with the above statistical analysis, activity appears restricted to the contralateral hemisphere at P10.

Figure 3 shows that a striking change occurs at P13; a short map with a focal positivity over the contralateral parietal cortex initiates the evoked response, preceding a second map with a focal negativity in the same area resembling the initial P7 and P10 maps. This new map has low amplitude and is not yet present in all animals (7 of 11). In agreement, a close look at the grand average waveforms (Figs. 1,2) reveals a small voltage-positive peak preceding the initial negative deflection at P13; however, it does not reach significant levels (Fig. 2). The third map resembles the late map observed at younger ages with respect to the positive potential at the parietal electrodes but shows additional negative potentials at the contralateral frontal electrode. 
At P16 and P21, activity invades wider areas in both hemispheres. The early focal positivity over the parietal cortex that was already partly observed at $\mathrm{P} 13$ is now fully developed, exhibiting strong field strength as indicated by the GFP in Figure 3 . The earliest positive map is present in all animals and appears at much shorter latencies than at P13 (ANOVA, $F_{(2,25)}=$ 185.5; $p<0.001$; Games-Howell tests at $p<0.001)$. Similar map configurations are observed at P16 and P21; however, the positive initial component appears at an earlier latency, at $\mathrm{P} 21$ rather than at P16 $(6.2 \pm 0.5 \mathrm{~ms}$ vs $8.3 \pm 0.7 \mathrm{~ms} ; p<0.001)$, and is now stronger in GFP amplitude than the second component map $(p<$ 0.01 ). The topographical analysis also reveals more components with distinct topographies in later time windows. At approximately $25 \mathrm{~ms}$ poststimulus at $\mathrm{P} 16$ and $20 \mathrm{~ms}$ at P21, the initial positivity reverses to a negative potential in the parietal area, while positive potentials invade the frontal area of the same hemisphere, as well as the parietal area of the other hemisphere (maps 2). During the third maps, polarities reverse in the hemisphere contralateral to the stimulation, while positive voltages are observed in the parietofrontal regions of the other hemisphere, which then reverse to negative in the following maps.

\section{Developmental shift at P13}

Because the most prominent developmental changes seemed to occur around P13 with the appearance of a new early component, we recorded epicranial SEPs in three animals successively at P12, P13, and P14. At P12, the evoked SEP at the contralateral parietal electrode begins with a negative voltage deflection in all three animals (Fig. $4 a, b$ ). At P13, a small positive deflection precedes the negative component in two of three animals and, as illustrated by an example in Figure $4 a$, the epicranial maps exhibit evoked voltages in the frontal region. Finally at P14, the SEP starts with an initial positive deflection in the contralateral parietal region of all three rats. At this age, evoked activity clearly invaded the hemisphere ipsilateral to the stimulus in all animals, which was not the case at P12.

Correlation between the development of the initial component and propagation of activity

The above results point to a crucial developmental stage at age P13, which is characterized by the appearance of an initial scalppositive potential in the contralateral parietal region and subsequent activation of a large-scale sensorimotor network that involves both hemispheres. To probe for direct relations between these two observations, we performed correlation analysis in P13 animals between the early positive activation at the $\mathrm{S} 1$ electrode and the total poststimulus absolute activations at the
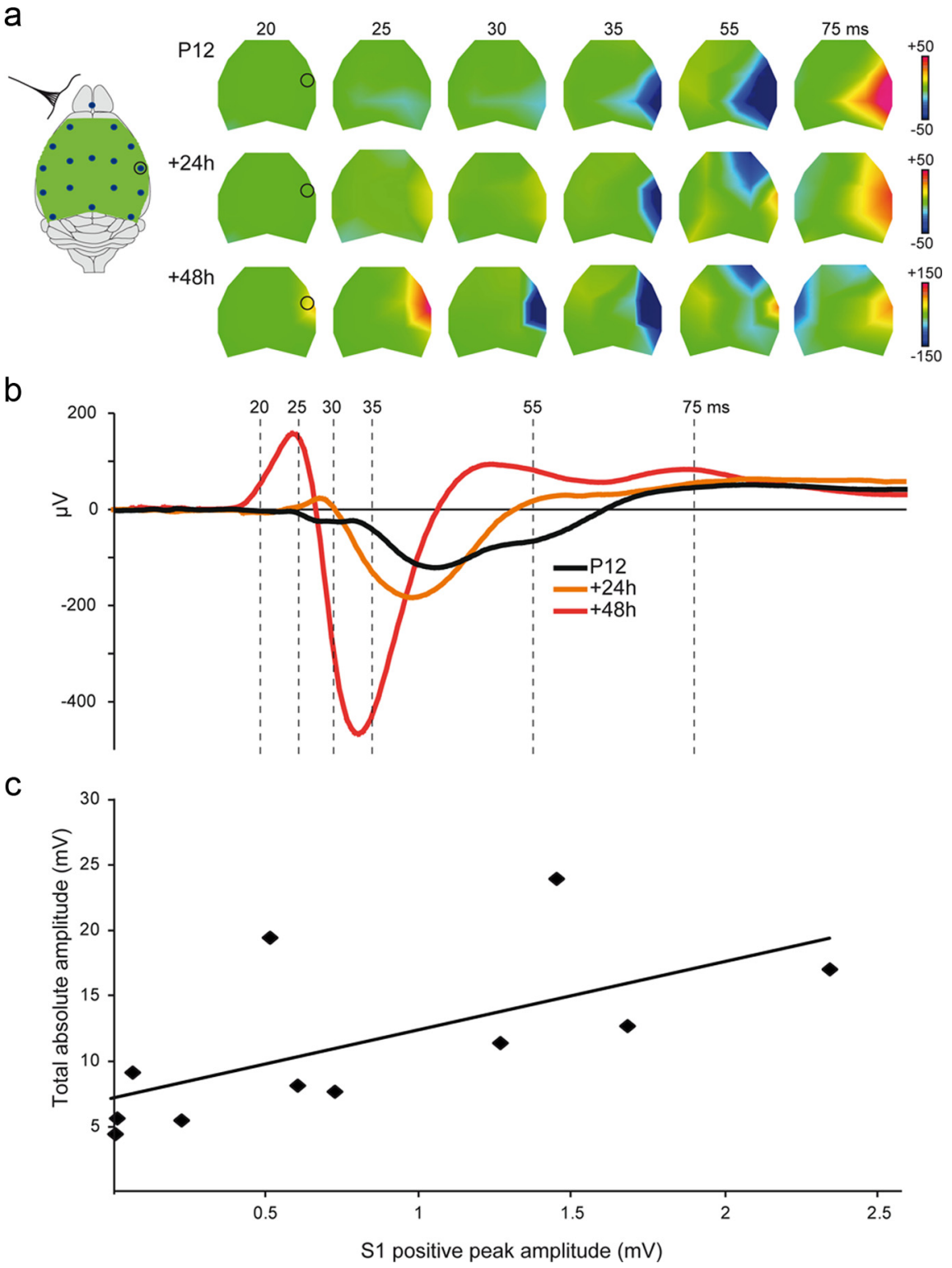

Figure 4. A short-latency positive component appears between P12 and P14. $\boldsymbol{a}$, Voltage maps at selected time points in esponse to left-sided whisker deflections recorded in the same animal at P12, P13, and P14. Color scaling in microvolts is indicated simulus (open circles in $\boldsymbol{a}$ ). c, Correlation analysis in all P13 animals $(n=11)$ between the early parietal positive peak amplitude, calculated as the sum of the voltages recorded at the $\$ 1$ electrode at each time bin across the duration of the positive component, and the total absolute activations at the electrodes located in the frontal area and in the parietal and frontal areas of the other hemisphere summated from 20 to 100 ms poststimulus. The linear regression line is illustrated (regression coefficient $=0.64$ ).

electrodes located in the frontal area and in the parietal and frontal areas of the other hemisphere (Fig. $4 c$ ). This analysis revealed a significant positive correlation between the parietal positive peak and the propagation of activity (linear regression model, $p=0.03)$. On the contrary, no correlation was found at P13 between the amplitude of the parietal negative peak and the total absolute activation at other electrodes $(p=0.49)$. Finally, it is important to recall here that at P16, once the positive parietal component is fully developed, the activations of the remote regions occur sooner than or simultaneously with the onset of the negative parietal component as indicated by the multiple statistical tests (Fig. 2), confirming the dependence of the propagation of activity on the early S1-positive component. 
a

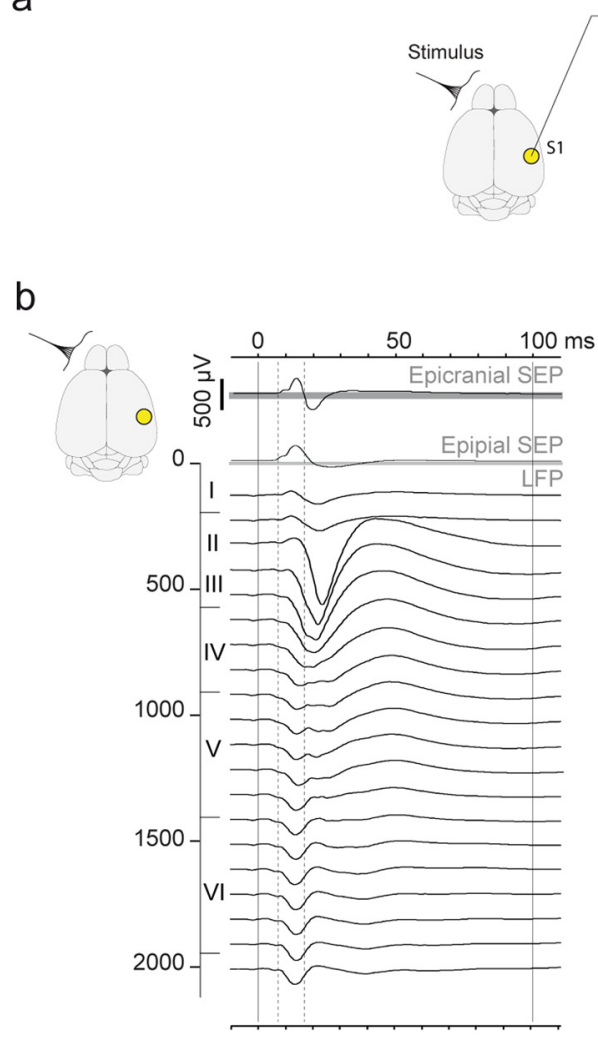

C

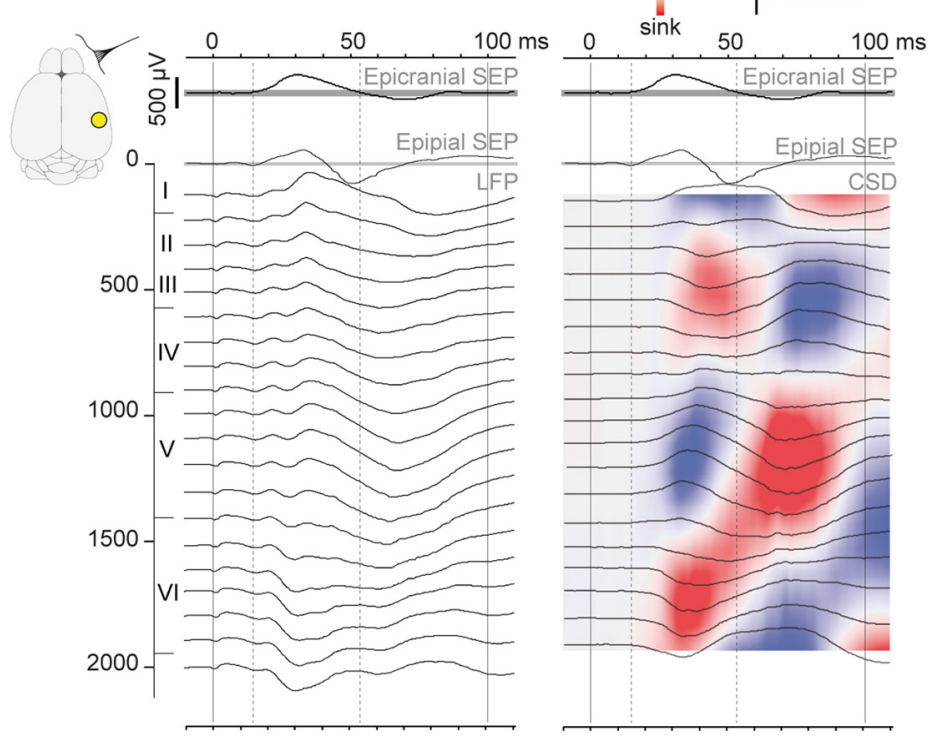

Figure 5. Recordings of the laminar activity profiles and CSD analyses. $\boldsymbol{a}$, Design of the intracortical recording setup. To study sensory evoked processing in contralateral and ipsilateral cortices, recordings were made in the right hemispheres with 16-electrode probes in response to left (contralateral) and right (ipsilateral) whisker stimulations. Electrode positions are illustrated on the photomicrograph of a Niss-stained coronal section of S1. The cortical thickness was sampled in two successive overlapping recordings allowing us to record 500 $\mu \mathrm{m}$ deeper (gray dots; see Materials and Methods). The epipial electrode (red) was not included in the CSD calculation. b, c, Epicranial, epipial, and intracortical recordings in response to contralateral (b) and ipsilateral (c) whisker stimulations in the S1 area of a P21 animal. The left panels show the intracortical LFP and the right panels the corresponding CSD traces, superimposed on color-coded plots of the interpolated CSD values. To ease comparisons between surface and deep recordings, dashed lines were placed at the onset and offset of the initial epicranial positive components determined directly on the waveforms. On they-axis are indicated cortical depths in micrometers and cortical layer borders.

Intracortical recordings in parietal and frontal areas

In the lissencephalic rat brain, it is likely that the cortical areas located beneath the focal maxima or minima of the epicranial SEP maps are the intracortical sources of the recorded surface maps (Mitzdorf, 1985; Mégevand et al., 2008). Epicranial mapping indicates that four cortical regions are implicated in the sensorimotor network: the parietal S1 cortices and the frontal cortices, containing the M1 vibrissa primary motor representation in the adult rat (Brecht et al., 2004; Chakrabarti et al., 2008), contralateral and ipsilateral to the stimulation. To confirm our surface data and to gain insight into the maturation of intracortical circuits of the sensorimotor network, we performed intracortical LFP recordings and CSD analysis in $\mathrm{P} 10$ and $\mathrm{P} 21$ rats in those four cortical regions.

Figure 5 displays an example of simultaneously recorded epipial and intracortical LFP and CSD profiles acquired in the S1 cortex of a P21 rat, together with the local epicranial SEPs recorded in the same rat. Epicranial and epipial electrodes both integrate cortical activity from two close but different viewpoints; accordingly, their traces are similar, but not identical. This might reflect the fact that the more distant epicranial electrode integrates cortical activity from a larger area. The filtering properties of the meninges and skull also likely play a role in explaining these differences. The epipial traces are also comparable but not identical to the LFP in the uppermost cortical layers. Because of volume conduction, it is difficult to infer the laminar localization of cortical activity from the LFP; however, the polarity reversal at the layer III/layer IV border during the initial surface component suggests the presence of local current generators. CSD analyses of the LFP allow increase of spatial resolution regarding the location of LFP generators and removal of far field contributions (Mitzdorf and Singer, 1977). CSD profiles confirm the presence, during the positive surface component, of a large current sink centered on layer IV, extending to upper layer V and lower layer III, which reflects the main excitatory drive of the thalamocortical projections (Agmon and Connors, 1992). This sink is balanced by a source in more superficial layers. The current sink then moves toward the supragranular layers, in parallel to a decrease in the surface component positivity, finally replaced by a negative component. In a markedly different pattern from that observed in response to contralateral stimulations, responses to ipsilateral stimulations start with two current sinks found in supragranular and infragranular layers.

Figure 6 presents the grand average CSD computed in the S1 and M1 cortices of 8 P10 and 8 P21 animals. At P10, intracortical evoked responses are almost completely restricted to the contralateral S1 cortex. Evoked CSD activity begins at $22.5 \pm 0.5 \mathrm{~ms}$ 

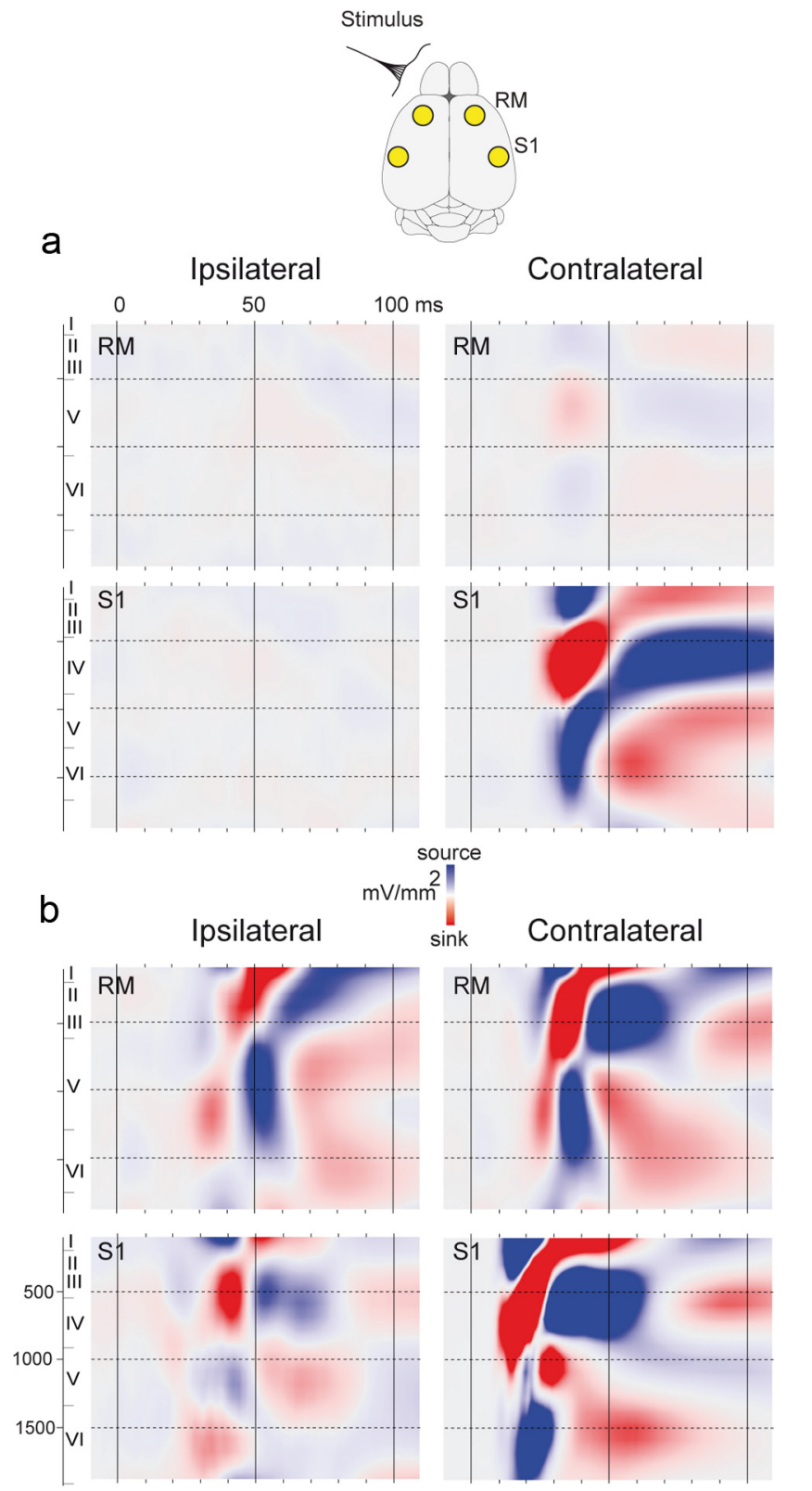

Figure 6. Current source-density analysis of intracortical responses to whisker stimulation at P10 and P21. $\boldsymbol{a}, \boldsymbol{b}$, Grand average current-source densities of 8 animals at P10 ( $\boldsymbol{a})$ and 8 animals at P21 $(\boldsymbol{b})$ processed in the frontal and S1 regions in response to contralateral and ipsilateral stimulations. The color-coded scale is $\pm 80 \mathrm{mV} / \mathrm{mm}^{2}$. The $y$-axis conventions are as in Figure 5 .

onset, with a current sink in contralateral S1 at 500-900 $\mu \mathrm{m}$ depth, corresponding to layer IV, flanked by superficial and deep sources (Fig. 6). This configuration progressively shifts toward the surface and then reverses to a layer IV source surrounded by two sinks after $60 \mathrm{~ms}$ poststimulus. Weak CSD activity was also observed in the contralateral M1 cortex, but was barely seen on the colored voltage plots, with a sink-source pattern similar to that in the $S 1$ cortex but $>20$ times weaker in amplitude.

At P21, evoked activity is observed in S1 and M1 of both hemispheres. Responses begin in contralateral S1 at $7.0 \pm 0.5 \mathrm{~ms}$ with a large current sink at 900-1100 $\mu \mathrm{m}$ depth, encompassing lower layer IV and upper layer $V$, rapidly spreading from 500 to $1200 \mu \mathrm{m}$ and balanced by a single current source in superficial layers. A large current source then develops $\sim 15 \mathrm{~ms}$ poststimulus in lower layer V and layer VI, while the current sink climbs up toward the cortical surface. Evoked activity in the M1 cortex contralateral to the stimulation starts at $18.5 \pm 0.5 \mathrm{~ms}$ onset with a current source $300-700 \mu \mathrm{m}$ in depth and a current sink 1000 $1500 \mu \mathrm{m}$ in depth both reversing $\sim 30 \mathrm{~ms}$ poststimulus. In $\mathrm{S} 1$ and M1 cortices ipsilateral to the stimulation, significant CSD activities appear as soon as 19 ms poststimulus, predominantly developing after $25 \mathrm{~ms}$ poststimulus, with complex sinksource configurations. In ipsilateral S1, sinks are initially found in supragranular and infragranular layers, while the response in ipsilateral M1 starts with a sink of $\sim 1200 \mu \mathrm{m}$, a configuration not unlike that of contralateral M1.

How does intracortical activity link with epicranial recordings? Local intracortical activity and epicranial SEPs showed obvious spatiotemporal correspondences, confirming that intracortical sources lay beneath the focal maxima and minima of the surface maps. Evoked intracortical activity was indeed present in areas where absolute maximal amplitudes of the epicranial SEP were observed, i.e., the contralateral S1 in P10 rats and the S1 and M1 of both hemispheres in P21 animals, while to the contrary, it was absent in the ipsilateral hemisphere of $\mathrm{P} 10$ rats, where no significant epicranial responses were recorded. Moreover, the temporal sequences of sink-source configuration changes match the reversals from focal maxima to focal minima of the SEP at corresponding epicranial sites. For instance, in the contralateral S1 cortex of P10 animals, the layer IV sink and its two neighboring sources all reverse after $60 \mathrm{~ms}$ poststimulus (Fig. 6), while epicranial SEPs recorded in this area showed a shift from a local minimum to a local maximum at similar latency (Fig. 3). At P21, the layer IV-layers II/III sink-source dipole that initiates the response in S1 (Fig. 6) reverses $\sim 20$ ms poststimulus when a deep source develops, matching the change from a local parietal epicranial maximum to a local minimum at similar latency (Fig. 3). Similarly, in the M1 cortex, the initial sink-source configuration reverses at $\sim 30 \mathrm{~ms}$, a period at which epicranial frontal activity also exhibited polarity reversion.

The most striking feature of the postnatal SEP maturation is the development of an initial positive component in the contralateral S1 area while, in immature animals, the initial component is negative. We therefore analyzed in more detail the intracortical currents evoked by whisker stimulus in the location of the probable generators of these negative and positive components at P10 and at P21, asking the question as to how sink-source configurations reflect the changes observed in epicranial recordings. We performed multiple paired $t$ tests with Bonferroni corrections comparing prestimulus to poststimulus CSDs across all animals within the P10 and P21 groups to reveal the dominant laminar currents (Fig. 7). At P10, significant evoked activity spreads in almost the entire cortical depth at $\sim 25 \mathrm{~ms}$ poststimulus and corresponds to a current sink in layer IV, flanked by superficial and deep sources. At comparable latencies, the grand average epicranial SEP recorded in the same animals shows a local negativity. At P21, the early SEP component is positive, and at similar latencies, the intracortical response pattern displays a significant sink from upper layer IV to lower layer III, balanced by a source in the upper layers. A deep source could be observed in several animals, but it did not reach significance with our statistical methods. Thus, current flows appear predominant in layer IV and supragranular layers during the initial surface positive component at the end of the postnatal period.

\section{Maturation of laminar activity}

Current sinks and sources originate from local changes in neuronal membrane conductance that mainly reflect synaptic currents 
a

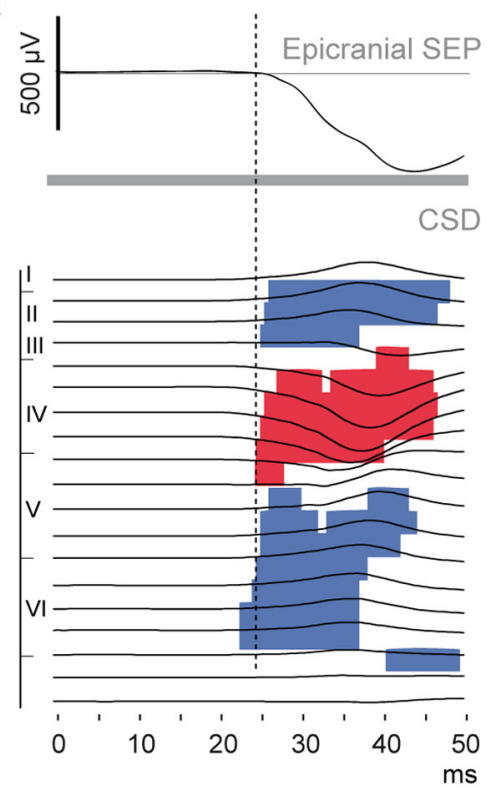

b

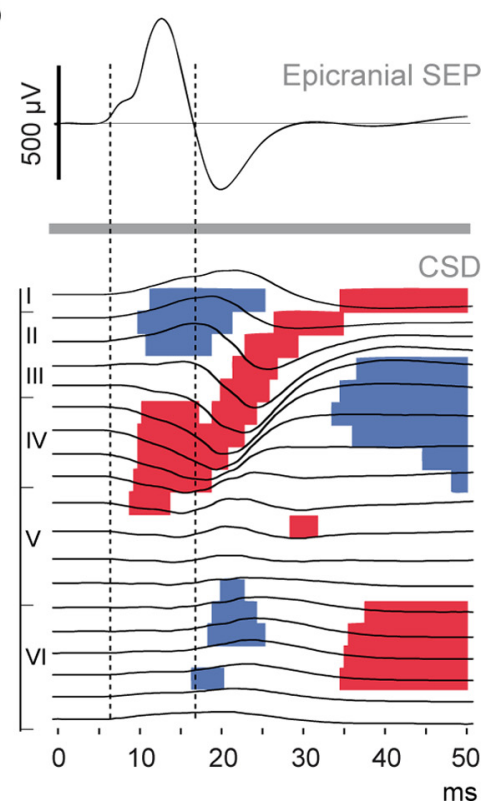

Figure 7. Correlation between epicranial SEPs and intracortical CSDs in S1 at P10 and P21. $\boldsymbol{a}$, $\boldsymbol{b}$, Colored segments indicate periods of significant evoked CSD signal at $p<0.01$ at each electrode in P10 (a) and P21 $(\boldsymbol{b})$ rats, superimposed on the CSD traces (point-wise paired $t$ tests with Bonferroni corrections). Above each panel are presented the grand average SEPs recorded in the same rats (P10,n=8;P21,n=8). Dashed lines point to onsets and offsets of the firstSEP components approximate from the grand average waveforms. The $y$-axis indicates the cortical layer borders.

and, much less, the rapidly propagating action potentials (Mitzdorf, 1985). We next completed the developmental profiles of cortical maturation by performing MUA analyses, which allow us to gain an insight into the laminar localization of the spike output (Fig. 8). These analyses show that in the S1 cortex of immature P10 animals, the main loci of MUA reside predominantly in layer IV, in correspondence with a strong local sink, and to a lesser extent in lower layer V and layer VI. Almost no MUAs were detected in supragranular layers. At P21, the laminar pattern of MUA has profoundly changed, starting with high MUA power in layer IV-lower layer III as well as in lower layer V, and then rapidly invading the entire layers II/III. Other MUA loci later appear in upper layer $\mathrm{V}$ and in layer VI. For illustrative purposes we have saturated the CSD color-coded plots (Fig. $8 d, f$ ); one can observe that MUA tends to correspond to current sinks, although MUA could also be observed in layers dominated by current sources, as in layer VI, for instance. The MUA power dramatically decreases in the entire cortical thickness as early as $20 \mathrm{~ms}$ poststimulus onset, which corresponds to the end of the S1 positive surface component described above (Fig. 3).

\section{Discussion}

Our results revealed the postnatal functional development of the sensorimotor large-scale cortical networks in rats during the second postnatal week. Until P10, cortical response is restricted to the parietal region where the contralateral S1 is located. At approximately $\mathrm{P} 13$, a new initial component with positive polarity develops above the sensory area, and evoked activity invades wider regions. After the second week, cortical processing between distant networks is established with evoked responses invading several cortical networks downstream of S1 in the frontal motor region and in the sensory and motor regions of the other hemisphere.

Sensory evoked responses can be elicited in the rat $\mathrm{S} 1$ cortex as early as P1 (Armstrong-James, 1975; McCandlish et al., 1993); however, as in newborns of other mammals comprising humans, cortical responses are weaker and slower than in adults (Pihko and Lauronen, 2004; Borgdorff et al., 2007). In agreement, we observed strong reinforcement and drastic acceleration of corti$\mathrm{cal}$ responses during the postnatal period. Responses start in the contralateral S1 area with a large current sink in layer IV, presumably generated by depolarization of the cortical neurons targeted by TC terminals already functionally connected during the first days of life (Catalano et al., 1991; Tenke et al., 1993; Peterson et al., 1995; Molnár et al., 2003; Khazipov et al., 2004; Kichula and Huntley, 2008). Decreasing onset latency with age reflects the maturation of subcortical (Shoykhet and Simons, 2008) and TC pathways including the myelination process, which is almost undetectable during the first week but increases rapidly during the second and third weeks (Fields, 2005; Jito et al., 2008).

Until P10, responses were mostly restricted to the contralateral parietal region where S1 is located. Weak intracortical activity was also observed in the frontal M1 cortex, but it was too weak to show on the epicranial recordings. Starting nearly at the same latency as and with source-sink distribution similar to that of S1, we assume that this activity is due to direct thalamocortical inputs to the motor cortex (Cicirata et al., 1986; Chakrabarti and Alloway, 2006) rather than to corticocortical projections from S1. From P16, evoked responses invade sequentially the parietal sensory and frontal motor regions of the contralateral and then ipsilateral hemispheres. Activation of the M1 cortex begins with a current sink in infragranular layers that should reflect feedforward activation from S1 projections to the motor cortex, since these were shown to activate predominantly its deeper layers (Hoffer et al., 2005; Chakrabarti et al., 2008). Several routes interconnecting S1 to the other hemisphere were demonstrated in rodents: direct projections between homotopic regions in parietal (Olavarria et al., 1984; Welker et al., 1988; Koralek et al., 1990) and frontal (Mitchell and Macklis, 2005) cortices, as well as polysynaptic paths between cortical areas including transcallosal corticothalamic projections (Molinari et al., 1985; Alloway et al., 2008). The infragranular sinks recorded in the ipsilateral hemisphere at P21 should reflect activation from callosal projections.

Interconnections between distant cortical networks are not fully formed at P10, which may explain the immaturity of their 
functional assembly. The maturation of callosal and frontal projections from sensory areas indeed span the entire postnatal period (Wise and Jones, 1978; Ivy and Killackey, 1982; Wang et al., 2007). Local circuitry in S1 is also immature and could be unable to generate efficient outputs toward downstream networks. In cortical layers II/III, the P10-P15 period is characterized by highly motile spines, huge increases in synaptic density and whiskerevoked synaptic potentials, and strong capacity for receptive field plasticity (Micheva and Beaulieu, 1996; Lendvai et al., 2000; Stern et al., 2001; Bureau et al., 2004). Most interestingly for our observations, the long-range cortical projections from S1 originate from neurons aligned with the layer IV septae in supragranular layers (Alloway et al., 2004; Chakrabarti et al., 2008), and these septal circuits were shown to maturate around P12-P16 (Bureau et al., 2004).

Along with the recruitment of downstream cortical networks, the most striking change we observed during the P10P15 period was the development of a new, early scalp positive component in the S1 area. The absence of this component before P13 is most likely not due to differences in sensitivity to steady-state adaptation (see Materials and Methods) or to different susceptibility to anesthesia in younger animals, because deepening anesthesia at P13 or later does not suppress it or alter its onset latency (data not shown). Absence of sensitivity to anesthesia of the early S1 response was also observed in adult mice (Mégevand et al., 2008). Rather, this new component points to an intrinsic difference in the way the S1 cortex processes sensory information and suggests the development of a new fast activation with S1 maturation. We show that at P21, the timing of this component corresponds to a main sink-source dipole from layer IV to superficial layers. This is distinctly different from the initial scalp negative potential at P10 that is characterized by a strong sink in layer IV flanked by two sources, one in the superficial and one in the profound layers. Since CSD analysis alone does not allow distinguishing between local synaptic activity and passive currents generated by branches of neurons (Mitzdorf and Singer, 1977), the interpretation of these different source-sink distributions is not straightforward. We therefore performed MUA analyses that confirm the profound differences between immature and mature animals: at P10, MUA remains restricted to layer IV and infragranular layers, while at P21, it invades further supragranular layers. Thus, the new positive surface component that develops in S1 constitutes a landmark of the maturation of local cortical processing. Furthermore, it correlates with the development of distant areas' activation in the sensorimotor network that relies on corticocortical connections leaving from supragranular layers. Future studies should address the exact nature of the mechanisms underlying the establishment of a new early component in S1. More generally, the rodent model could help to better understand the cortical generation of surface components, allowing b
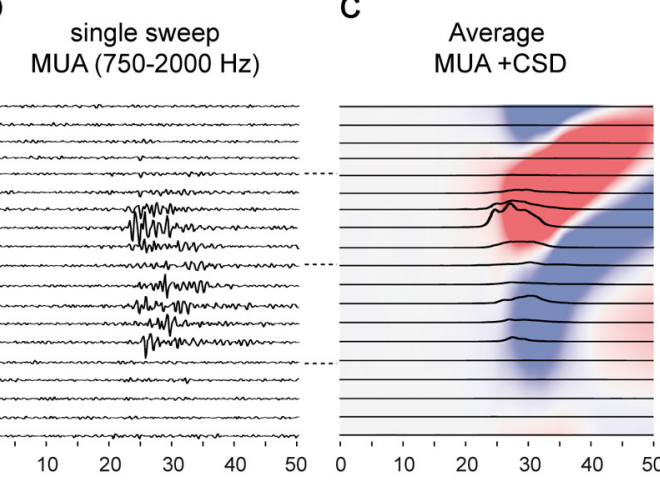

f

e

single sweep MUA $(750-2000 \mathrm{~Hz})$
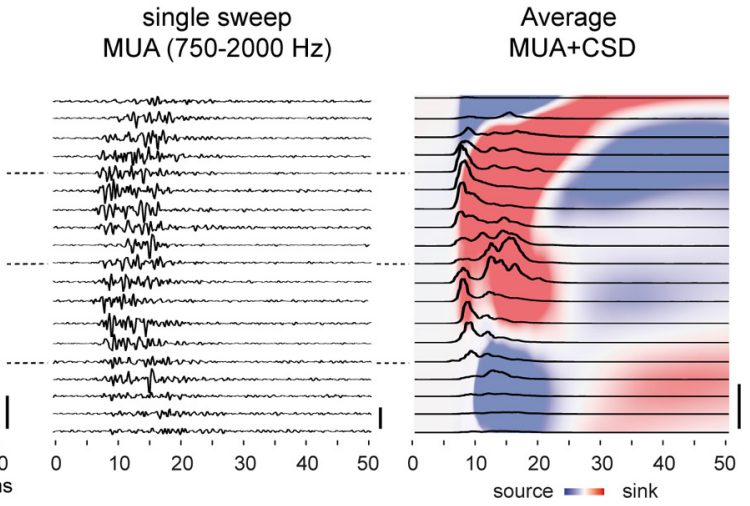

Average

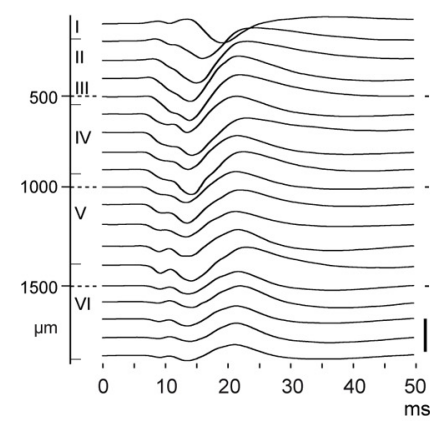

Figure 8. Laminar LFP and MUA in the $S 1$ cortex at $\mathrm{P} 10$ (top row) and at $\mathrm{P} 21$ (bottom row). $\boldsymbol{a}, \boldsymbol{b}$ and $\boldsymbol{d}, \boldsymbol{e}$, Example voltage traces in a P10 and a P21 rat, respectively, recorded in response to a single sweep contralateral whisker deflection and showing the low , calibration, $1 \mathrm{mV}$ )- and high (MUA without rectification; calibration, $50 \mu \mathrm{V}$ )-frequency components of the poststimulus one to manipulate neuronal activity at the cellular and molecular levels in a lissencephalic cortex.

The maturation of cortical processing we observed around P13 corresponds not only to a period of profound transformations of the synaptic network in S1 (Micheva and Beaulieu, 1996; Lendvai et al., 2000; Stern et al., 2001; Bureau et al., 2004) but also to the initiation of whisking exploratory behaviors (Carvell and Simons, 1990). Sensory evoked activity during this critical period at the end of the second postnatal week is crucial for the development of layer II/III somatosensory maps (Stern et al., 2001) and may also operate in the maturation of sensorimotor networks. Self-sustained cortical activity as well as activity evoked by spontaneous muscular movements could also play a role (Khazipov et al., 2004; Price et al., 2006). The neonatal rat cortex and, interestingly, that of the premature human baby exhibit spontaneous activity patterns characterized by spindle-like bursts in the $5-25 \mathrm{~Hz}$ frequency range spatially confined to local cortical areas (Khazipov and Luhmann, 2006; Golshani et al., 2009). It has been proposed that these high synchronicity patterns result from intrinsic synaptic properties of excitatory neurons and immature inhibition, and could serve cortical map maturation by strengthening or eliminating synapses between coactive neurons (Voutsinos-Porche et al., 2003; Golshani et al., 2009). In the barrel cortex, the transition from the highly correlated state to the more mature decorrelated state also occurs at the end of the second postnatal week (Golshani et al., 2009). 
Based on cytoarchitecture, the $\mathrm{P} 10-\mathrm{P} 15$ period in rats corresponds to late preterm to early full-term human neonates (Clancy et al., 2007). The best described feature in the development of SEPs in premature babies is the shortening of the first cortical response N1 (for review, see Vanhatalo and Lauronen, 2006), which corresponds to a unilateral fronto-occipital potential gradient created by a tangential dipole in the postcentral gyrus (Karniski, 1992). Developmental changes of later components were also described in premature babies; however, the underlying cortical events are difficult to infer because of the paucity of topographical SEP data, the lack of intracortical recordings, and the gyrification process that could modify the orientation of cortical currents. In full-term neonates, recordings of somatosensory evoked magnetic fields during the first month of life also showed the development of new components (Pihko et al., 2009), but after the initial arrival of the information in $\mathrm{S} 1$, while our data indicate a new component at the very beginning of the arrival in S1 and thus a new distribution of synaptic activity within the cortical columns of S1.

Future studies should define the developmental processes of large-scale cortical networks by investigating functional synaptic connections between single neurons in distant networks. The functional approach we used to analyze network development in the rodent cortex also offers opportunities to study the impact of signaling pathways and damaging insults on specific developmental events in defined cortical subregions.

\section{References}

Agmon A, Connors BW (1992) Correlation between intrinsic firing patterns and thalamocortical synaptic responses of neurons in mouse barrel cortex. J Neurosci 12:319-329.

Ahissar E, Kleinfeld D (2003) Closed-loop neuronal computations: focus on vibrissa somatosensation in rat. Cereb Cortex 13:53-62.

Ahissar E, Sosnik R, Haidarliu S (2000) Transformation from temporal to rate coding in a somatosensory thalamocortical pathway. Nature 406:302-306.

Alloway KD, Zhang M, Chakrabarti S (2004) Septal columns in rodent barrel cortex: functional circuits for modulating whisking behavior. J Comp Neurol 480:299-309.

Alloway KD, Olson ML, Smith JB (2008) Contralateral corticothalamic projections from MI whisker cortex: potential route for modulating hemispheric interactions. J Comp Neurol 510:100-116.

Armstrong-James M (1975) The functional status and columnar organization of single cells responding to cutaneous stimulation in neonatal rat somatosensory cortex S1. J Physiol 246:501-538.

Armstrong-James M, Fox K (1987) Spatiotemporal convergence and divergence in the rat S1 "barrel" cortex. J Comp Neurol 263:265-281.

Benison AM, Rector DM, Barth DS (2007) Hemispheric mapping of secondary somatosensory cortex in the rat. J Neurophysiol 97:200-207.

Borgdorff AJ, Poulet JF, Petersen CC (2007) Facilitating sensory responses in developing mouse somatosensory barrel cortex. J Neurophysiol 97:2992-3003.

Brecht M, Krauss A, Muhammad S, Sinai-Esfahani L, Bellanca S, Margrie TW (2004) Organization of rat vibrissa motor cortex and adjacent areas according to cytoarchitectonics, microstimulation, and intracellular stimulation of identified cells. J Comp Neurol 479:360-373.

Bureau I, Shepherd GM, Svoboda K (2004) Precise development of functional and anatomical columns in the neocortex. Neuron 42:789-801.

Carvell GE, Simons DJ (1990) Biometric analyses of vibrissal tactile discrimination in the rat. J Neurosci 10:2638-2648.

Catalano SM, Robertson RT, Killackey HP (1991) Early ingrowth of thalamocortical afferents to the neocortex of the prenatal rat. Proc Natl Acad Sci U S A 88:2999-3003.

Chakrabarti S, Alloway KD (2006) Differential origin of projections from SI barrel cortex to the whisker representations in SII and MI. J Comp Neurol 498:624-636.

Chakrabarti S, Zhang M, Alloway KD (2008) MI neuronal responses to peripheral whisker stimulation: relationship to neuronal activity in SI barrels and septa. J Neurophysiol 100:50-63.
Cicirata F, Angaut P, Cioni M, Serapide MF, Papale A (1986) Functional organization of thalamic projections to the motor cortex. An anatomical and electrophysiological study in the rat. Neuroscience 19:81-99.

Clancy B, Darlington RB, Finlay BL (2001) Translating developmental time across mammalian species. Neuroscience 105:7-17.

Clancy B, Finlay BL, Darlington RB, Anand KJ (2007) Extrapolating brain development from experimental species to humans. Neurotoxicology 28:931-937.

Dagnew E, Latchamsetty K, Erinjeri JP, Miller B, Fox K, Woolsey TA (2003) Glutamate receptor blockade alters the development of intracortical connections in rat barrel cortex. Somatosens Mot Res 20:77-84.

Erzurumlu RS, Jhaveri S (1990) Thalamic axons confer a blueprint of the sensory periphery onto the developing rat somatosensory cortex. Brain Res Dev Brain Res 56:229-234.

Ferezou I, Haiss F, Gentet LJ, Aronoff R, Weber B, Petersen CC (2007) Spatiotemporal dynamics of cortical sensorimotor integration in behaving mice. Neuron 56:907-923.

Fields RD (2005) Myelination: an overlooked mechanism of synaptic plasticity? Neuroscientist 11:528-531.

Foeller E, Feldman DE (2004) Synaptic basis for developmental plasticity in somatosensory cortex. Curr Opin Neurobiol 14:89-95.

Fox K (2002) Anatomical pathways and molecular mechanisms for plasticity in the barrel cortex. Neuroscience 111:799-814.

Golshani P, Gonçalves JT, Khoshkhoo S, Mostany R, Smirnakis S, PorteraCailliau C (2009) Internally mediated developmental desynchronization of neocortical network activity. J Neurosci 29:10890-10899.

Hoffer ZS, Arantes HB, Roth RL, Alloway KD (2005) Functional circuits mediating sensorimotor integration: quantitative comparisons of projections from rodent barrel cortex to primary motor cortex, neostriatum, superior colliculus, and the pons. J Comp Neurol 488:82-100.

Ivy GO, Killackey HP (1982) Ontogenetic changes in the projections of neocortical neurons. J Neurosci 2:735-743.

Jito J, Nakasu S, Ito R, Fukami T, Morikawa S, Inubushi T (2008) Maturational changes in diffusion anisotropy in the rat corpus callosum: comparison with quantitative histological evaluation. J Magn Reson Imaging 28:847-854.

Karniski W (1992) The late somatosensory evoked potential in premature and term infants. I. Principal component topography. Electroencephalogr Clin Neurophysiol 84:32-43.

Khazipov R, Luhmann HJ (2006) Early patterns of electrical activity in the developing cerebral cortex of humans and rodents. Trends Neurosci 29:414-418.

Khazipov R, Sirota A, Leinekugel X, Holmes GL, Ben-Ari Y, Buzsáki G (2004) Early motor activity drives spindle bursts in the developing somatosensory cortex. Nature 432:758-761.

Kichula EA, Huntley GW (2008) Developmental and comparative aspects of posterior medial thalamocortical innervation of the barrel cortex in mice and rats. J Comp Neurol 509:239-258.

Kleinfeld D, Ahissar E, Diamond ME (2006) Active sensation: insights from the rodent vibrissa sensorimotor system. Curr Opin Neurobiol $16: 435-444$.

Koralek KA, Olavarria J, Killackey HP (1990) Areal and laminar organization of corticocortical projections in the rat somatosensory cortex. J Comp Neurol 299:133-150.

Lendvai B, Stern EA, Chen B, Svoboda K (2000) Experience-dependent plasticity of dendritic spines in the developing rat barrel cortex in vivo. Nature 404:876-881.

McCandlish CA, Li CX, Waters RS (1993) Early development of the SI cortical barrel field representation in neonatal rats follows a lateral-to-medial gradient: an electrophysiological study. Exp Brain Res 92:369-374.

Mégevand P, Quairiaux C, Lascano AM, Kiss JZ, Michel CM (2008) A mouse model for studying large-scale neuronal networks using EEG mapping techniques. Neuroimage 42:591-602.

Michel CM, Murray MM, Lantz G, Gonzalez S, Spinelli L, Grave de Peralta R (2004) EEG source imaging. Clin Neurophysiol 115:2195-2222.

Micheva KD, Beaulieu C (1996) Quantitative aspects of synaptogenesis in the rat barrel field cortex with special reference to GABA circuitry. J Comp Neurol 373:340-354.

Miller B, Blake NM, Erinjeri JP, Reistad CE, Sexton T, Admire P, Woolsey TA (2001) Postnatal growth of intrinsic connections in mouse barrel cortex. J Comp Neurol 436:17-31.

Mitchell BD, Macklis JD (2005) Large-scale maintenance of dual projec- 
tions by callosal and frontal cortical projection neurons in adult mice. J Comp Neurol 482:17-32.

Mitzdorf U (1985) Current source-density method and application in cat cerebral cortex: investigation of evoked potentials and EEG phenomena. Physiol Rev 65:37-100.

Mitzdorf U, Singer W (1977) Laminar segregation of afferents to lateral geniculate nucleus of the cat: an analysis of current source density. J Neurophysiol 40:1227-1244.

Molinari M, Minciacchi D, Bentivoglio M, Macchi G (1985) Efferent fibers from the motor cortex terminate bilaterally in the thalamus of rats and cats. Exp Brain Res 57:305-312.

Molnár Z, Kurotani T, Higashi S, Yamamoto N, Toyama K (2003) Development of functional thalamocortical synapses studied with current sourcedensity analysis in whole forebrain slices in the rat. Brain Res Bull 60:355-371.

Olavarria J, Van Sluyters RC, Killackey HP (1984) Evidence for the complementary organization of callosal and thalamic connections within rat somatosensory cortex. Brain Res 291:364-368.

Peterson NN, Schroeder CE, Arezzo JC (1995) Neural generators of early cortical somatosensory evoked potentials in the awake monkey. Electroencephalogr Clin Neurophysiol 96:248-260.

Pihko E, Lauronen L (2004) Somatosensory processing in healthy newborns. Exp Neurol 190 [Suppl 1]:S2-S7.

Pihko E, Nevalainen P, Stephen J, Okada Y, Lauronen L (2009) Maturation of somatosensory cortical processing from birth to adulthood revealed by magnetoencephalography. Clin Neurophysiol 120:1552-1561.

Price DJ, Kennedy H, Dehay C, Zhou L, Mercier M, Jossin Y, Goffinet AM, Tissir F, Blakey D, Molnár Z (2006) The development of cortical connections. Eur J Neurosci 23:910-920.

Quairiaux C, Sizonenko SV, Mégevand P, Michel CM, Kiss JZ (2010) Functional deficit and recovery of developing sensorimotor networks following neonatal hypoxic-ischemic injury in the rat. Cereb Cortex 20:2080-2091.

Rash BG, Grove EA (2006) Area and layer patterning in the developing cerebral cortex. Curr Opin Neurobiol 16:25-34.

Schlaggar BL, O'Leary DD (1994) Early development of the somatotopic map and barrel patterning in rat somatosensory cortex. J Comp Neurol 346:80-96.
Sehara K, Toda T, Iwai L, Wakimoto M, Tanno K, Matsubayashi Y, Kawasaki H (2010) Whisker-related axonal patterns and plasticity of layer $2 / 3$ neurons in the mouse barrel cortex. J Neurosci 30:3082-3092.

Shoykhet M, Simons DJ (2008) Development of thalamocortical response transformations in the rat whisker-barrel system. J Neurophysiol 99:356-366.

Shuler MG, Krupa DJ, Nicolelis MA (2001) Bilateral integration of whisker information in the primary somatosensory cortex of rats. J Neurosci 21:5251-5261.

Stern EA, Maravall M, Svoboda K (2001) Rapid development and plasticity of layer 2/3 maps in rat barrel cortex in vivo. Neuron 31:305-315.

Sur M, Leamey CA (2001) Development and plasticity of cortical areas and networks. Nat Rev Neurosci 2:251-262.

Tenke CE, Schroeder CE, Arezzo JC, Vaughan HG Jr (1993) Interpretation of high-resolution current source density profiles: a simulation of sublaminar contributions to the visual evoked potential. Exp Brain Res 94:183-192.

Troncoso E, Muller D, Czellar S, Zoltan Kiss J (2000) Epicranial sensory evoked potential recordings for repeated assessment of cortical functions in mice. J Neurosci Methods 97:51-58.

Vanhatalo S, Lauronen L (2006) Neonatal SEP. Back to bedside with basic science. Semin Fetal Neonatal Med 11:464-470.

Voutsinos-Porche B, Knott G, Tanaka K, Quairiaux C, Welker E, Bonvento G (2003) Glial glutamate transporters and maturation of the mouse somatosensory cortex. Cereb Cortex 13:1110-1121.

Wang CL, Zhang L, Zhou Y, Zhou J, Yang XJ, Duan SM, Xiong ZQ, Ding YQ (2007) Activity-dependent development of callosal projections in the somatosensory cortex. J Neurosci 27:11334-11342.

Welker C (1976) Receptive-fields of barrels in somatosensory neocortex of rat. J Comp Neurol 166:173-189.

Welker E, Hoogland PV, Van der Loos H (1988) Organization of feedback and feedforward projections of the barrel cortex: a PHA-L study in the mouse. Exp Brain Res 73:411-435.

Wise SP, Jones EG (1978) Developmental studies of thalamocortical and commissural connections in the rat somatic sensory cortex. J Comp Neurol 178:187-208. 